1 Dominance rank predicts social network position across developmental stages

\title{
in rhesus monkeys
}

$4 \quad{ }^{1}$ Eunice Kennedy Shriver National Institute for Child Health and Human Development, National

14 Running title: Social rank predicts social network position 


\section{Abstract}

Social network analysis is increasingly common in studying the complex interactions

22 among individuals. Across a range of primates, high-ranking adults are generally more socially

23 connected, which results in better fitness outcomes. However, it still remains unclear whether

24 this relationship between social network position and dominance rank emergences in infancy and

25 whether, in species with a social transmission of dominance rank, social network positions are

26 driven by the presence of the mother. To fill this gap, we first explored whether dominance ranks

27 were related to social network position, measured via eigenvector centrality, in infants, juveniles,

28 and adults in a troop of semi-free ranging rhesus macaques (Macaca mulatta). We then

29 examined relationships between dominance rank and eigenvector centrality in a peer-only group

30 of yearlings who were reared with their mothers in either a rich, socially complex environment of

31 multigenerational (MG) kin support or a unigenerational (UG) group of mothers and their infants

32 from birth through eight months. In experiment 1, we found that mother's network position

33 predicted offspring network position, and that dominants across all age categories were more

34 central in affiliative networks (social contact, social grooming, and social play). Experiment 2

35 showed that high-ranking yearlings in a peer-only group were more central only in the social

36 contact network. Moreover, yearlings reared in a socially complex environment of MG kin

37 support were more central. Our findings suggest that the relationship between dominance rank

38 and social network position begins early in life, and that complex early social environments can

39 promote later social competency. Our data add to the growing body of evidence that the

40 presence/absence of the mother and kin influence how dominance rank affects social network

41 position. These findings have important implications for the role of caregivers in the social status

42 of developing primates, which ultimately ties to health and fitness outcomes. 
43 Keywords: eigenvector centrality, dominance rank, development, social network, rhesus

44 macaques

\section{Introduction}

Primates have a slow developmental trajectory, with maturation into adulthood requiring several years (Harvey \& Clutton-Brock, 1985; Pereira \& Fairbanks, 2002). Several hypotheses

49 suggest that primate evolution has favored an extended juvenile period as a way for individuals

50 to better learn a variety of skills needed for survival including proper foraging strategies

51 (Agostini \& Visalberghi, 2005; Janson \& van Schaik, 1993; Rapaport \& Brown, 2008), specific

52 mechanisms to avoid predators (Ramakrishnan \& Coss, 2001; Seyfarth, Cheney, \& Marler,

53 1980), and necessary social skills needed to integrate into the social group (Joffe, 1997; Shimada

$54 \&$ Sueur, 2014). Given the vast array of challenges that developing primates must successfully

55 overcome, it is perhaps not surprising that juveniles may be especially susceptible to mortality

56 (Ross \& Jones, 1999). While several studies have explored how young primates learn foraging

57 and predator avoidance strategies (Hauser, 1988; Rapaport \& Brown, 2008; van de Waal,

58 Claidiére, \& Whiten, 2013), investigations on how infants develop their social skills to better

59 integrate into the group's social networks are still scant (Barale, Rubenstein, \& Beehner, 2015;

60 Kulik, Amici, Langos, \& Widdig, 2015). Yet, understanding how individuals acquire their social

61 competence from infancy can shed new light on how social integration affects both human and

62 nonhuman primate survival, health, and overall fitness across the lifespan (Brent, Ruiz-

63 Lambides, \& Platt, 2017; Kroenke, Kubzansky, Schernhammer, Holmes, \& Kawachi, 2006;

64 Lehmann, Majolo, \& McFarland, 2015; Smith \& Christakis, 2008; Vogt, Mullooly, Ernst, Pope, $65 \&$ Hollis, 1992). 
Among all mammalian taxa, primates are one of the groups with the highest levels of

67 sociality, reflected by a remarkable degree of gregariousness and an extraordinary variety of

68 social systems (Kappeler \& van Schaik, 2002). Many primate species, thus, engage in a complex

69 network of social connections, and accumulating evidence indicates that individuals' fitness is

70 affected not only by their direct social relationships, but also by their indirect social ties (e.g., if

71 they are connected with individuals who have also a lot of social connections; Brent, 2015).

72 Social network analysis is an increasingly common tool to analyze these elaborate ties (both

73 direct and indirect) among individuals (Brent, 2015; Farine \& Whitehead, 2015; McCowan,

74 Anderson, Heagarty, \& Cameron, 2008; McCowan et al., 2016; Pinter-Wollman et al., 2014;

75 Sueur, Jacobs, Amblard, Petit, \& King, 2011). Social network analysis has been used to study a

76 wide variety of outcomes, ranging from disease transmission (Griffin \& Nunn, 2011; MacIntosh

77 et al., 2012; Rushmore et al., 2013) to social learning (Coelho et al., 2015; Hobaiter, Poisot,

78 Zuberbühler, Hoppitt, \& Gruber, 2014), and captive primate welfare (McCowan et al., 2008).

79 However, to date, the vast majority of the research on nonhuman primate social networks has

80 focused on adults, due either to the assumption that adult social networks are long established

81 and therefore stable; to difficulties in recognizing juveniles and infants, especially as they are

82 constantly changing and growing; or simply to the study questions at hand which may not

83 warrant immature subjects. Therefore, although we are beginning to learn more about social

84 networks in adults, relatively little is known about the social networks of developing primates.

85 Yet, recent work suggests that examining the developmental trajectory of infant and juvenile

86 networks can be key to understanding social networks in adults.

First, recent studies have highlighted that adult social networks are not always stable over 
89 Vandeleest et al., 2017). Therefore, understanding the extent of changes in individuals' network

90 positions and social ties across their lifespan is pivotal to have a better understanding of how

91 these changes can reflect different fitness outcomes. Second, a handful of studies on both primate

92 and non-primate species have shown that juveniles provide a meaningful contribution to the

93 group social network as a whole, and their exclusion can significantly impact the assessment of a

94 group network structure (e.g., olive baboons, Papio Anubis: Fedurek \& Lehmann, 2017; orcas,

95 Orcinus orca: Williams \& Lusseau, 2006).

One potential mechanism that could relate to a juvenile's position in social networks is

97 dominance rank. For primate species that live in large social groups, dominance rank is a

98 pervasive factor in behavioral development. For example, across a variety of primate species,

99 high-ranking individuals are preferred grooming partners, as dominants can offer low-ranking

100 individuals rank-restricted services, such as tolerance and agonistic support, in exchange for

101 grooming (Carne, Wiper, \& Semple, 2011; Seyfarth, 1977). Consequently, dominance ranks are

102 expected to influence an individual's position in the social network. Accordingly, across some

103 primate species, high-ranking adults occupy more central (i.e., more socially connected)

104 positions in their social networks (Borgeaud, Sosa, Sueur, \& Bshary, 2017; Sueur \& Petit, 2008;

105 Sueur et al., 2011), and this relationship appears particularly strong in despotic species that

106 display low levels of affiliation, more asymmetric aggression, steep dominance relationships, and

107 low rates of post-conflict reconciliation (Sueur et al., 2011).

In primates characterized by social transmission of dominance rank, such as in

109 cercopithecines (Berman, 1980; Cheney, 1977), a potential mechanism by which infants and

110 juveniles can acquire their social network position may be through kin support and influence in

111 their social interactions. In other words, if high-ranking mothers or kin are central in their social 
112 networks and support their offspring in acquiring high ranks, then their offspring are expected to

113 develop more central positions in the network as well. To date, however, evidence of maternal or

114 kin influence of juveniles' acquisition of network positions has been limited. Berman,

115 Rasmussen, \& Suomi (1997) found, for instance, that rhesus macaque (Macaca mulatta) mothers

116 in large groups bias their infant's interactions towards close kin, suggesting that the mother can

117 have a significant impact on infant social networks. Turner, Bills, and Holekamp (2018) found

118 that social networks vary based on rank in developing hyenas (Crocuta crocuta), a species

119 characterized by maternal rank inheritance, but they did not directly test whether mother's

120 presence or behavior influenced their offspring's social network position. Interestingly, Jarrett et

121 al., (2018) found that juvenile vervet (Chlorocebus pygerythrus) networks did not map onto their

122 mother's networks (which may have been explained by temporal shifts in the mothers'

123 networks), although they did conclude that mother-daughter associations may influence both of

124 their associations with others. Therefore, it still remains unclear: (1) whether the relationship

125 between dominance rank and social network position emergences in infancy and juvenility and

126 (2) whether this relationship, if present, is due to mothers' (and other close kin) presence, which

127 may influence an infant's interactions with other group members.

The goals of this study were therefore two-fold: (1) we first examined whether

129 dominance rank predicted an individual's position in its social networks in both immature

130 (newborns to 2 years) and mature (3+ years) rhesus macaques in a naturalistic population (with

131 mothers and multigenerational (MG) kin support; experiment 1); (2) we then explored the

132 relationship between dominance rank and social network position in yearling rhesus macaques

133 living in a peer-only group without the presence of adult kin, including their mothers

134 (experiment 2). Rhesus macaques provide the ideal study model to address these questions. They 
135 are female philopatric (i.e., females remain in their natal group while males emigrate at puberty),

136 and are characterized by a highly nepotistic (i.e., social interactions are largely directed towards

137 close kin) and despotic (i.e., frequent aggression and a linear, asymmetric dominance hierarchy)

138 social system (Thierry, 2007). Rhesus monkey dominance rank is correlated with social network

139 position, with top-ranking females being more central in the network (Sueur et al., 2011), and is

140 acquired by a hearty system of matrilineal support in aggressive interactions (Berman, 1980),

141 resulting in offspring 'inheriting' the rank of their mothers.

142 In experiment 1, we examined the development of the relationship between dominance

143 rank and social network position in a naturalistic, MG population of rhesus macaques from

144 infancy through adulthood. We first sought to replicate Sueur et al. (2011)'s results, predicting

145 that high-ranking adults would be more central in their social networks (prediction 1). Similarly,

146 if social network position is potentially inherited, then we predicted that mothers' social network

147 position should be correlated with their offspring's network position (prediction 2) and that in

148 infants and juveniles, like in their mothers, social network position would be related to

149 dominance rank (prediction 3). In experiment 2, we examined the relationship between

150 dominance rank and social network position in a yearling group of rhesus macaques recently

151 formed after having been reared in social groups with their mothers for the first eight months of

152 life. This peer group was formed by taking nine yearlings from the MG (mothers, siblings, aunts,

153 grandmothers, great-grandmothers, cousins, etc.) field station and nine yearlings from

154 unigenerational (UG; only same-aged mothers and their infants) groups and combining them into

155 one social group, without the mothers. This yearling group was studied both immediately after

156 group formation and hierarchy establishment, and following group relocation to a familiar

157 environment for the UG subjects. This relocation resulted in a complete reversal of the hierarchy 
158 and a period of prolonged social instability (Wooddell, Kaburu, Murphy, Suomi, \& Dettmer,

159 2017a), since the UG subjects challenged the previously dominant MG subjects. In Wooddell et

160 al. (2017a) we argued that this rank reversal is likely to be related to UGs' familiarity with the

161 environment, as previous work across a broad range of social species has shown that familiarity

162 with the environment can predict dominance rank (e.g., dark-eyed juncos, Junco hyemalis:

163 Cristol \& Ketterson, 1990; willow tit, Parus montanus: Koivula, Lahti, Orell, \& Rytkönen,

164 1993). If factors other than kin support affect offspring's social network position, (e.g.,

165 dominance rank), then high-ranking yearlings should be more central in their social networks

166 following the formation of the peer-only group (prediction 4), and this should hold following a

167 complete reversal of the hierarchy (i.e., previous low-ranked yearlings should now be more

168 central; prediction 5).

\section{Materials and Methods}

170 All procedures here and below adhered to the NIH Guide for the Care and Use of

171 Laboratory Animals, the American Society of Primatologists Principles for the Ethical Treatment

172 of Nonhuman Primates, and were approved by the NICHD Animal Care and Use Committee.

\section{Subjects and housing}

\section{Experiment 1}

176 at the Laboratory of Comparative Ethology field station in Poolesville, MD. The troop was a

177 large, MG group formed in the 1980s that consisted of three matrilines. The dominance ranks of

178 the matrilines in order were: matriline 3 , matriline 4 , and matriline 1 , with the exception of the

179 alpha male, who was from matriline 1 (the lowest-ranking matriline). It is important to note that 
180 the numbers of the matrilines do not refer to ordinal rank, but rather to the laboratory's historical

181 naming method for the matrilines. Matriline 2 was removed in 2004 due to management reasons.

182 Extensive matrilineal relationships have been documented in a previous publication (Wooddell,

183 Kaburu, Suomi, \& Dettmer, 2017b). The troop lived in a semi-naturalistic enclosure and

184 exhibited the naturalistic social structure and dominance attainment classic of other free and

185 semi-free ranging rhesus macaque groups. Subjects lived in a 5-acre (2-ha) wooded outdoor

186 open-air enclosure with natural vegetation, foliage, and a pond (0.9 ha) with an island (0.07 ha),

187 in addition to various enrichment such as jungle gyms, swings, perches, and tree huts. Three

188 corncrib shelters $(4.88 \times 4.88 \times 5.79 \mathrm{~m})$ and three indoor runs $(2.74 \times 5.79 \times 4.27 \mathrm{~m})$ provided

189 protection from inclement weather, but subjects had ad libitum access to all areas of the habitat

190 (indoor and outdoor), unless for cleaning purposes. Purina Monkey Chow (\#5038, St. Louis,

191 MO), natural vegetation, and water were available ad libitum and a mixture of seeds, nuts, and

192 fruit were provided twice a day. Subjects were exposed to both ambient light and temperature in

193 the outdoor portion, but the indoor portions were set on a $12: 12$ light cycle at $25.6^{\circ} \mathrm{C}$.

\section{Experiment 2}

Subjects in experiment 2 were 18 rhesus macaque yearlings (born in 2015; 14 males; four

196 females) studied from January to August 2016 following the formation of a peer group

197 (Wooddell et al., 2017a). Briefly, nine of these subjects (six males, three females) were

198 previously reared in one of three UG groups from birth in 2015 to January 2016. The UG groups

199 consisted of 10-12 same-aged adult females, one adult male, and 1-4 several same-aged infants.

200 The UG groups lived in indoor $(2.44 \times 3.05 \times 2.21 \mathrm{~m})$ and outdoor $(2.44 \times 3.0 \times 2.44 \mathrm{~m})$

201 enclosures equipped with perches, wood shavings, hanging barrels and were fed Purina High

202 Protein Monkey Chow (\#5045, St. Louis, MO) and fresh fruit and vegetables twice a day. The 
203 outdoor portions were exposed to ambient light and temperature, and the indoor portions were on

204 a 12:12 light cycle and constant temperature of $25.6^{\circ} \mathrm{C}$. The other nine subjects (eight males, one

205 female) were previously reared in the MG group in the field station from birth in 2015 and were

206 nine (of the 18) of the infants in experiment 1. These subjects were removed in December 2015

207 and January 2016 following a rare overthrow among the adult females in the troop at the field

208 station, in which previously lower-ranking females attacked the dominant females and upended

209 the social hierarchy (Wooddell et al., 2017b). In January 2016, these 18 yearlings (nine from the

210 UG groups and nine from the MG field station group) were combined to create a peer-only

211 group, in addition to one unrelated adult "grandfather" male. The group lived in a novel

212 environment of an indoor run $(7.3 \times 3.4 \times 3.7 \mathrm{~m})$ with ad libitum access to an outdoor corncrib

213 (5.03 in diameter by $5.49 \mathrm{~m}$ high) from January to May 30, 2016. On May 31, 2016, the group

214 was relocated to the rearing environment of the UG subjects (see above for description on

215 housing details). Due to the familiarity of the environment for the UG (but not MG subjects), this

216 relocation prompted a series of unstable dominance relationships, with an eventual complete

217 reversal of the dominance hierarchy (see Wooddell et al., 2017a). The group resided in the

218 indoor-outdoor runs familiar to the UG subjects until August 2016 when the study ended.

\section{Behavioral data collection: social affiliation}

220 For experiment 1, LJW collected data from 36 adult subjects (4 males, 32 females; ages

221 3-18 years, $\mathrm{M} \pm \mathrm{SD}: 8.19 \pm 3.34$ years), 25 juvenile subjects (11 males, 14 females, age at the

222 beginning of the study: 1-2 years; 404-904 days: 602.36 \pm 169.13 days), and 18 infant subjects

223 (14 males, four females; age at the beginning of the study: 31-64 days; $45 \pm 10.29$ days). Infants

224 were distinguished from juveniles by age, as infants were heavily dependent on the mother,

225 being only a month or two old at the start of the study, whereas juveniles were relatively 
226 independent and were well over a year (or two) old at the start of the study. Adult behavioral

227 data were collected 1-2 times per week in both AM (900-1200) and PM sessions (1200-1700) via

228 modified frequency sheets (Novak, Kinsey, Jorgensen, \& Hazen, 1998) from November 2014 to

229 July 2015 using a 5-minute continuous focal animal sampling method (Altmann, 1974). Each 5-

230 minute interval was broken into 20,15 -second intervals in which the frequencies of all behaviors

231 (social and nonsocial) were recorded in chronological order. For the purposes of this study, we

232 only analyzed social behaviors, which included social contact (i.e., within $30-\mathrm{cm}$ of another

233 individual), social grooming (i.e., picking and spreading apart the fur), and social play (i.e.,

234 rough and tumble wrestling, play chasing, open mouth play faces). For each social behavior, the

235 behaviors were mutually exclusive for a dyad (i.e., an individual was not in social contact with

236 the individual they were grooming, but they could be in social contact with a different

237 individual). For each of these behaviors, we also recorded the identities of the social partners to

238 construct social networks so as to analyze the number of intervals that focal animals interacted

239 with other individuals. A total of 1,528 adult observations were recorded ( $M=42.44 \pm 9.43)$,

240 totaling 127.33 hours. Behavioral data on juveniles and infants were recorded in both AM and

241 PM sessions following the identical coding scheme as the adult behavioral collection. For

242 juveniles, data were collected twice per week from September to December 2015. A total of 728

243 juvenile observations $(\mathrm{M}=29.12 \pm 1.09)$ were recorded, totaling 60.67 hours. Behavioral data on

244 infants were recorded 2-3 times per week across both AM and PM sessions from June to

245 December 2015. The infants were born from March to May of 2015 so the observations reflected

246 approximately the first 3-8 months of life (or slightly earlier for the later born infant). A total of

$2471,067$ infant observations were recorded (59.28 \pm 4.32$)$, totaling 88.92 hours. Total observation

248 time for the field station was thus 277 hours. 
For the peer group in experiment 2, each yearling (age at the beginning of the study: 188-

250296 days: $274.44 \pm 24.85$ days) was observed twice per week in both AM and PM sessions by

251 one of three observers (inter-observer reliability $\geq 85 \%$ for at least 3 consecutive sessions),

252 following identical procedures as in experiment 1 . We collected a total of 615 observations

$253(\mathrm{M}=34.17 \pm 1.25)$ while the monkeys were housed in the new environment following peer group

254 formation, totaling 51.25 hours. Following relocation to the previous rearing environment of the

255 UG subjects in May 2016, and during subsequent hierarchical instability, 360 observations were

256 recorded $(M=20 \pm 0.34)$, totaling 30 hours. Total observation time for the peer group across both

257 housing conditions (and periods of decreasing stability) was thus 81.25 hours. It is important to

258 note that nine of the infants from the field station were eventually relocated to the peer-group

259 following the overthrow in the field station, thus they are included in both experiments (1 and 2).

260 These infants were therefore studied continuously from approximately three months of age to 1.5

261 years across different environments.

\section{Behavioral data collection: dominance hierarchies}

263 To construct dominance hierarchies, we used all observations of aggressive (threat, chase,

264 attack) and submissive (displacement, fear grimace) interactions recorded via both focal animal

265 sampling and ad libitum sampling (Altmann, 1974). For the field station, a total of 10,994

266 dominance interactions were recorded from November 2014 to December 2015 among all group

267 members (infants, juveniles, and adults). For the peer-group, a total of 5,835 dominance

268 interactions were recorded (after group formation: 2,604; after group relocation and instability:

269 3,231). We constructed dominance hierarchies via Elo-rating, a method commonly used to detect

270 temporal fluctuations in dominance rank over time (Neumann et al., 2011), which we have

271 previously utilized for both of the current study populations (Wooddell et al., 2017a; 2017b). For 
272 all experiments, we used the elo.sequence function (Neumann et al., 2011) in R software (version

273 3.3.3) with the initial value set at 1,000 and the $\mathrm{k}$ factor set at 200. An Elo-rating is generated for

274 both the winner and loser after each interaction, with more points attributed to the interaction in

275 which the outcome was unexpected (i.e., a lower-ranking animal winning against a higher-

276 ranking animal) than expected interactions. Higher Elo-ratings reflect higher dominance ranks.

277 Therefore, a total of 21,988 Elo-ratings were used to generate dominance ranks for experiment 1 ,

278 and 11,670 Elo-ratings were generated to determine dominance ranks for experiment 2. Average

279 Elo-ratings were used in analyses. It is important to note that we used the infant and juveniles'

280 own dominance ranks (instead of their mothers') based off of their dominance interactions,

281 which allowed us to examine both an inheritance of dominance rank and social network position.

282 Social network analysis

In experiment 1 , we analyzed only adult-adult interactions to construct adult social

284 networks. To construct peer networks, we used juvenile-juvenile interactions (1-2 years old) to

285 construct juvenile social networks, and only infant-infant interactions to construct infant social

286 networks. We chose to examine three types of affiliative social networks independently - social

287 contact, social grooming, and social play networks - as each of these networks can provide

288 different social mechanisms depending on age. For example, social grooming is primarily done

289 by adult females, and can serve to strengthen and maintain bonds (Dunbar, 1991) necessary for

290 group cohesion (Lehmann, Korstjens, \& Dunbar, 2007) and coalition formation (Borgeaud \&

291 Bshary, 2015; Schino, 2007). Social play on the other hand, is done by primarily immature

292 primates, especially males, and serves to maintain bonds (Poirier \& Smith, 1974), and possibly

293 assess rivals (Paquette, 1994), and promote social competence (Palagi, 2018; Pellis, Burghardt,

294 Palagi, \& Mangel, 2015). Social contact likely has similar functions across all ages. For adults, 
295 we constructed two types of networks: social contact and social grooming. We could not analyze 296 adult social play networks because play was never recorded between adult dyads. Contrarily, for 297 juveniles, we analyzed all three affiliative networks. And finally, for infants, we analyzed only

298 social contact and social play networks, because social grooming between infant dyads was only 299 observed once. In experiment 2, we used all peer interactions in addition to interactions with the 300 adult male, but excluded the adult male from statistical analysis. We constructed all three types 301 of affiliative networks for this peer group in experiment 2. For all experiments, we used 302 weighted, undirected networks for all affiliative networks.

304 Butts, Goodreau, \& Morris, 2006) in R software (version 3.3.3). We used the 'evcent' function to 305 calculate eigenvector centrality for each individual and for each social behavior. This network 306 metric reflects the number and strength of an individual's direct connections as well as the 307 centrality of the neighbors the subject is connected to (Bonacich, 2007; Kasper \& Voelkl, 2009; 308 Newman, 2004; Farine \& Whitehead, 2015; Sueur et al., 2011). In other words, individuals can 309 have high eigenvector centrality values either if they have a higher number of connections, or if 310 their neighbors have a higher number of connections, or both (Farine \& Whitehead, 2015).

311 Eigenvector centrality is a common metric analyzed in social networks and has previously been 312 reported to be associated with dominance rank in adult rhesus macaques (Sueur \& Petit, 2008;

313 Sueur et al., 2011). For visual illustrations of the social networks, we used Cytoscape (v 3.5.1).

\section{Development of a rank-related relationship}

315 To examine when a possible relationship emerged between dominance rank and 316 eigenvector centrality in infancy, we additionally combined and analyzed the social data into 4317 week bins. Given that the infants were studied for 29-weeks, the last bin contained 5-weeks. For 
318 each 4-week bin, we combined all affiliative data (social contact, social grooming, and social

319 play) among peers in order to obtain enough data for network construction and constructed social

320 networks as above. We then ran simple Spearman correlations to test whether Elo-ratings were

321 correlated with eigenvector centrality in each 4-week bin to analyze when this relationship

322 emerged.

\section{Statistical analysis}

In order to assess whether adult dominance rank predicted adult social network position

325 (prediction 1), we used the ' $\mathrm{lm}$ ' function in R to run two regression models (one for social

326 contact and one for social grooming). To this end, we set eigenvector centrality as the dependent

327 variable, while individuals' Elo-rating values, age and sex were set as predictors. We square-root

328 transformed social contact eigenvector values in order to normalize their distribution.

329 In order to assess whether mothers' social network predicts their offspring's social

330 network position (prediction 2), we ran a linear mixed model analysis (LMM) with the 'lmer'

331 function implemented in the R package lmerTest. Offspring's social play eigenvector was set as

332 the outcome variable. We set the mothers' social grooming and social contact eigenvector

333 centrality measures, infants' sex and age, and the interactions between infants' sex and mothers'

334 grooming and social contact eigenvectors as predictors. In this model, each mother's ID was

335 included as random factor in order to control for pseudoreplications due to a single mother

336 birthing multiple offspring ( $n=43$ offspring, $n=30$ unique mothers).

337 To test prediction 3 (i.e., that dominance rank also predicts social network position in 338 infants and juveniles), we ran multiple regression models via the ' $1 \mathrm{~m}$ ' function where eigenvector 339 values from social contact, social grooming (juveniles only), and social play were set as outcome 
340 variables. Infants' and juveniles' Elo-rating, sex, age, and their interaction were set as predictors.

341 For this analysis, juvenile social grooming and social play eigenvector values and infant social

342 play eigenvector centrality values were squared-root transformed, while both juvenile and infant

343 social contact eigenvector centrality values were log-transformed to achieve normal distribution.

344 Furthermore, for the analysis conducted on infant networks, an interaction between sex and

345 mother's Elo-rating was not tested due to a strongly male-biased sex ratio (14 males, four 346 females).

Finally, in order to test the relationship between yearling's rank and social network

348 position in the peer-only groups (predictions 4 and 5), we ran an LMM model where the social

349 contact, social grooming, and social play eigenvector values were included as dependent

350 variables in separate models, whereas early social experience (MG vs UG), sex, age, Elo-rating,

351 and period (before vs after relocation) were set as fixed factors. IDs were included as random

352 factors.

For all models, we took an information theoretic (I-T) approach to model selection and 354 selected only the best set of candidate models on the basis of AIC scores (Burham et al, 2011).

355 We did this by using the function 'stepAIC' implemented in the R package Mass for the 356 regression models and the function 'step' in the R package lmerTest for the LMM models. Only 357 the results from the best models (i.e., those with the lowest AIC scores) are presented in the 358 tables.

Network metrics are not independent because each individual's network metric (e.g., 360 eigenvector centrality) inevitably depends on other individuals' position in the network. This 361 lack of independency violates some of the assumptions of many statistical tests. In order to take 362 this into account, test statistics originated from observed data need to be compared with a 
363 distribution of test statistics generated from random networks (Farine, 2017). In the current

364 study, for each of the above-mentioned models (regression and LMMs), we generated 100

365 networks where we randomly swapped the identity of the recipient in the social interactions, and

366 then ran the models (regressions or LMMs) where eigenvector centrality values calculated from

367 each of these random networks were set as outcome variables. We then generated a distribution

368 of estimates from the models and examined where the estimate calculated from the observed data

369 falls in relation to such distribution. In order to calculate whether the regression and LMM

370 analyses from the observed data significantly differ from the analysis calculated from random

371 networks, we calculated one-tailed $\mathrm{p}$ values by comparing the number of the random estimates

372 that were higher than the observed estimate.

\section{Results}

374 Experiment 1: Relationship between social network position and dominance rank across the

375 lifespan in a naturalistic population

376 Adult eigenvector values ranged from 0.026 to 0.338 (M \pm SD: $0.15 \pm 0.07$ ) for social

377 contact and 0.01 to $0.36(0.147 \pm 0.08)$ for social grooming. Social contact and social grooming

378 eigenvector values were positively correlated for adults (Pearson correlation: $\mathrm{r}=0.46, \mathrm{p}=0.005$,

$379 \mathrm{n}=36$ ). The best model with grooming eigenvector as the outcome variable explained almost $60 \%$

380 of its variation $\left(\mathrm{F}(1,34)=52.51, \mathrm{p}<0.001, \mathrm{R}^{2}=0.595\right)$, and contained exclusively adults' Elo-

381 rating, which had a significant positive effect on grooming eigenvector centrality $(\beta=0.00013$,

$382 \mathrm{SE}=0.00002, \mathrm{t}=7.247, \mathrm{p}<0.001$; Table 1 ). This suggests that, among adult rhesus macaques,

383 dominance rank significantly predicted grooming network position (Figure 1a). Similarly, our

384 analysis of social contact showed that both Elo-rating and sex were part of the best model, which

385 explained about $16 \%$ of the variation in individuals' social contact eigenvector values 
$386\left(\mathrm{~F}(2,33)=4.236, \mathrm{p}=0.023 \mathrm{R}^{2}=0.156\right)$. In this model, Elo-rating had a significant positive effect

387 on social contact eigenvector $(\beta=0.00009, \mathrm{SE}=0.00003, \mathrm{t}=2.714, \mathrm{p}=0.01$; Table 1$)$. Both

388 models were significantly different from a random distribution (Figures S1 and S2). This

389 indicates that high-ranking adults had a greater number of social connections than low-ranking

390 adults, supporting prediction 1.

When we tested prediction 2, we found that the best model included exclusively the

392 interaction between mothers' grooming eigenvector (but not mothers' social contact eigenvector)

393 and offspring (infants and juveniles) sex, which significantly explained $40 \%$ of the variance of

394 offspring play network eigenvector centrality $(\beta=2.45, \mathrm{SE}=0.83, \mathrm{t}=2.94, \mathrm{p}=0.005$, Table 2$)$.

395 This model was significantly different from a random distribution (Figure S3), and revealed that

396 a positive relationship between mother's grooming eigenvector centrality and offspring's play

397 eigenvector centrality is present only in males but not females (Figure 2).

Mother's Elo-rating positively predicted juvenile's Elo-ratings $(\mathrm{F}(1,23)=34.82, \mathrm{p}<0.001$,

$\left.399 \mathrm{R}^{2}=0.602, \beta=0.78\right)$, indicating an inheritance of dominance rank. Juvenile eigenvector values

400 ranged from 0.007 to $0.515(0.124 \pm 0.161)$ for social contact, 0 to $2.06(0.203 \pm 0.419)$ for

401 social grooming, and $0.001 \pm 0.479(0.14 \pm 0.146)$ for social play. Only social contact and social

402 play eigenvector values were positively correlated $(r=0.53, p=0.007, n=25)$. For social grooming,

403 only age predicted eigenvector centrality $(\beta=0.0008, \mathrm{SE}=0.0002, \mathrm{t}=3.29, \mathrm{p}=0.003$; Table 1$)$,

404 whereas for social contact, Elo-rating was part of the best model $(\beta=0.0002, \mathrm{SE}=0.0001, \mathrm{t}=2.22$,

$405 \mathrm{p}=0.036$; Table 1; which was also significantly different from a random distribution; see Figure

406 S4). This model explained about $14 \%$ of the variation in juvenile social contact eigenvector

$407\left(\mathrm{~F}(1,23)=4.911, \mathrm{p}=0.037, \mathrm{R}^{2}=0.140\right)$. For social play, the top model included an interaction

408 between Elo-rating and sex, which explained $43 \%$ of the variance $(F(3,21)=7.144, p=0.002$, 
$409 \mathrm{R}^{2}=0.434$ ) and was significantly different from a random distribution (Figure S3). Collectively,

410 these results indicate that while older juveniles were more central in social grooming networks,

411 more dominant juveniles were more central in social contact networks, and high-ranking juvenile

412 males had a greater number of well-connected social partners than low-ranking males (Figures

$4131 \mathrm{~b}$ and 3).

414 Infant eigenvector values ranged from 0.03 to $0.4998(0.185 \pm 0.151)$ for social contact

415 and 0.037 to $0.539(0.185 \pm 0.150)$ for social play, and both were positively correlated (Pearson

416 correlation: $\mathrm{r}=0.60, \mathrm{p}=0.008, \mathrm{n}=18$ ). Mother's Elo-rating positively predicted her infant's Elo-

417 rating $\left(\mathrm{F}(1,16)=29.14, \mathrm{p}<0.001, \mathrm{R}^{2}=0.65, \beta=0.80\right)$, indicating an inheritance of dominance rank.

418 The analysis of infant social contact network position showed that the best model explained 51\%

419 of social contact eigenvector variation $\left(\mathrm{F}(1,16)=, \mathrm{p}<0.001, \mathrm{R}^{2}=0.507\right)$ and included exclusively

420 infant Elo-rating $(\beta=0.002, \mathrm{SE}=0.00005, \mathrm{t}=4.30, \mathrm{p}<0.001$; Table 1). Similarly, the best model in

421 which social play eigenvector was set as the outcome variable explained $43 \%$ of its variation

$422\left(\mathrm{~F}(2,15)=, \mathrm{p}=0.006, \mathrm{R}^{2}=0.428\right)$, and included infant Elo-rating which had a significantly positive

423 effect on their network position $(\beta=0.0003, \mathrm{SE}=0.0001, \mathrm{t}=3.29, \mathrm{p}=0.005$; see Table 1 and

424 Figures 1c and 4). Both models were significantly different from a random distribution (Figures

425 S5 and S6). High-ranking infants thus had a greater number of well-connected social partners

426 than low-ranking infants. These results indicate that the relationship between eigenvector

427 centrality and dominance rank emerged early in life, supporting prediction 3 . We constructed 7 ,

428 4-week bins and ran simple Spearman correlations between infant Elo-rating and eigenvector

429 centrality. Within the first 4-weeks of data collection (representing the first 2-3 months of life

430 depending on age at the start of the study), a correlation already emerged $(r=0.49, p=0.047)$, and

431 remained steady for the first 4, 4-week bins (weeks 5-8 of data collection: $\mathrm{r}=0.482, \mathrm{p}=0.04$, 
432 weeks $9-12$ of data collection: $r=0.49, p=0.04$, weeks $13-16$ of data collection: $r=0.74, p<0.001)$.

433 The relationship between eigenvector centrality and infant Elo-rating was not significant in

434 weeks $17-20$ and 21-24 ( $\mathrm{p}=0.30$ and 0.09 respectively), but was again significant in weeks 25-29

$435(\mathrm{r}=0.54, \mathrm{p}=0.02)$. In other words, high-ranking infants had more developed social networks than

436 low-ranking infants very shortly after birth.

437 Experiment 2: Relationship between social network position and dominance rank in a newly

438 formed peer group

439 After yearling group formation and before group relocation, eigenvector values ranged

440 from 0.066 to $0.409(0.198 \pm 0.129)$ for social contact, 0.006 to $0.604(0.163 \pm 0.175)$ for social

441 grooming, and 0.057 to $0.344(0.219 \pm 0.089)$ for social play. After group relocation and social

442 instability, eigenvector values ranged from 0.129 to $0.322(0.222 \pm 0.060)$ for social contact,

4430.016 to $0.585(0.158+0.167)$ for social grooming, and 0.014 to $0.422(0.202+0.125)$ for social

444 play. Both before and after social instability, social contact and social grooming eigenvector

445 values were positively correlated to each other (before: $r=0.72, p=0.001$; after: $r=0.684$,

$446 \mathrm{p}=0.002$ ), but both social contact (before: $\mathrm{r}=-0.608, \mathrm{p}=0.007$; after: $\mathrm{r}=-0.660, \mathrm{p}=0.003$ ) and social

447 grooming eigenvector values (before: $r=-0.530, p=0.023$; after: $r=-0.518, p=0.028$ ) were

448 inversely correlated with social play eigenvector values. Interestingly, the negative correlation

449 between social contact and social play is in sharp contrast to what was found in the naturalistic

450 population, where a positive correlation between these two variables was found. For the social

451 contact network, the best model included yearling early social experience (MG or UG: $\beta=-0.14$,

$452 \mathrm{SE}=0.020, \mathrm{t}=-6.719, \mathrm{p}<0.001 ;$ Table 3$)$ and Elo-rating $(\beta=0.00010, \mathrm{SE}=0.000015, \mathrm{t}=$

453 6.591, $\mathrm{p}<0.001$; Table 3), and this model was significantly different from a random distribution

454 (Figure S7). In contrast, contrary to our predictions, Elo-rating did not predict yearling 
455 eigenvector values for social grooming and social play networks, as for both analyses, the best

456 models included early social experiences and sex (Table 3). Collectively, these results indicate

457 that only for the social contact network, high-ranking yearlings were more central than low-

458 ranking yearlings, but only during a specific period (partially supporting prediction 4, failing to

459 support prediction 5). Furthermore, eigenvector values after group formation were predictive of

460 eigenvector values after group relocation and instability (social contact: $F(1,16)=21.92, p<0.001$,

$461 R^{2}=0.58, \beta=0.76$; social grooming: $F(1,16)=51.68, p<0.001, R^{2}=0.76, \beta=0.87$; social play:

$\left.462 \mathrm{~F}(1,16)=10.74, \mathrm{p}=0.005, \mathrm{R}^{2}=0.40, \beta=0.63\right)$, indicating that network positions remained stable

463 over time, although dominance ranks changed significantly (see Wooddell et al., 2017a for the

464 change in dominance ranks over time).

465 Discussion

466 Similar to previous findings (Sueur \& Petit, 2008; Sueur et al., 2011), in experiment 1

467 high-ranking adult macaques were more central in both social contact and social grooming

468 networks. This is not surprising, given that high-ranking adults can provide benefits to others,

469 including tolerance and agonistic support in exchange for grooming (Carne et al., 2011; Seyfarth,

470 1977), making them attractive as social partners, even in groups of related females in which

471 maternal lineage and dominance rank are independent (Snyder-Mackler et al., 2016).

472 Unfortunately, we did not have a large enough sample size of adult males $(n=4)$ to test for sex

473 effects of social networks. However, some research suggests that high-ranking adult male

474 macaques are likely to be more central as well (Sade, Altmann, Loy, Hausfater, \& Breuggeman,

475 1988; Sueur \& Petit, 2008; Sueur, Petit, et al., 2011), confirming previous work showing that the

476 fitness consequences of strong social bonds are not just limited to females (males: Schülke,

477 Bhagavatula, Vigilant, \& Ostner, 2010; Young, Majolo, Heistermann, Schülke, \& Ostner, 2014; 
478 females: Brent et al., 2017; Silk et al., 2010; Silk, Alberts, \& Altmann, 2003). Future research

479 should continue to examine the social network positions of both males and females across the 480 lifespan.

482 her son's position in the peer social play network, indicating a potential inheritance of social 483 networks (see also: Brent et al., 2013; Goldenberg, Douglas-Hamilton, \& Wittemyer, 2016; Ilany 484 \& Akçay, 2016). The reason why a mother's position in the grooming network is related to her 485 son's position in the play network, rather than his grooming network, likely has to do with their 486 life-history stages and the relative importance of each social behavior in each stage of life. For 487 adults, grooming is a primary behavior to maintain bonds and form alliances (Borgeaud \& 488 Bshary, 2015; Dunbar, 1991; Lehmann et al., 2007; Schino, 2007), whereas social play can serve 489 these same functions for young primates, especially males (Palagi, 2018; Paquette, 1994; Pellis 490 et al., 2015; Poirier \& Smith, 1974). To the best of our knowledge, we are unaware of any study 491 examining whether infants exchange play for agonistic support, which is an exciting avenue for 492 future research. Therefore, the grooming network for adults may be functionally similar to the 493 play network for young primates. In this case, high-ranking juveniles may be attractive social 494 partners in play, much the same way as high-ranking adults are attractive partners in social 495 grooming. Alternatively, infants could play around their mothers while they are grooming one 496 another or play with close kin that share similar dominance ranks, resulting in similar networks 497 (Berman, 1982). A further alternative scenario might be that mothers could actively initiate or 498 prevent social interactions of their infants (Berman et al., 1997). Unfortunately, we were unable 499 to tease apart the direct mechanisms by which offspring inherit similar network positions as the 500 mother, which needs to be tested with future studies. 
Similarly, mothers' dominance ranks predicted the infants' position in their social

502 networks, and this emerged during the first four weeks of data collection. How might social

503 networks already be constructed around dominance rank at such an early age, when infants are

504 still heavily dependent on the mother? Our findings indicate that the networks are at least in

505 some way being influenced by the mother's presence soon after, if not immediately after, birth.

506 Although the networks between mothers and infants are somewhat independent (as the mothers'

507 networks contained only adults and the infant networks contained only infants), infants could

508 still be associating with similar individuals as their mothers (e.g., the infants of those mothers).

509 High-ranking infants may therefore be associating with a plethora of other individuals, simply

510 because their mother is. As the mother has a strong influence on the social networks of her

511 infants (Berman 1982, Berman et al., 1997; Maestripieri, 2018), future research should analyze

512 social interactions with or without the mother in close proximity. This will allow us to

513 investigate to what extent networks change depending on the mother's nearby association and

514 possible involvement.

515 In the naturalistic population, we found that high-ranking juvenile males were more

516 central in social play networks, but high-ranking females were not. This is at first surprising,

517 given that females remain in their natal groups, and males emigrate at puberty. Our findings

518 suggest that juvenile males may find additional benefits of integration into peer social networks

519 than females. For example, previous research has indicated that groups of same-aged (and often

520 related) male macaques may emigrate together (van Noordwijk \& van Schaik, 2001) and form

521 alliances with one another (de Ruiter \& Geffen, 1998; Gerber, Krützen, de Ruiter, van Schaik, \&

522 van Noordwijk, 2015), which can increase the chances of a successful integration, thereby

523 increasing reproductive fitness (Schülke et al., 2010). Strengthening peer relationships may 
524 therefore be an especially adaptive strategy for juvenile males. Moreover, it may be that males

525 may need to be socially 'primed' for dispersal. Indeed, some research indicates that nonhuman

526 primate mothers differentially invest in male offspring (for a review, see Lonsdorf, 2017): by

527 exhibiting more mutual gazing with sons after birth (Dettmer, Kaburu, Byers et al., 2016), which

528 may promote social competency (Dettmer, Kaburu, Simpson, et al., 2016), and by increasing

529 sons' social networks to include other mothers with offspring (Murray et al., 2014) and non-

530 matrilineal group members (Timme, 1995). By increasing the complexity of the social

531 experiences for male offspring, mothers may 'prime' males to be more socially savvy, with a

532 variety of individuals, which can have long-term fitness consequences. Data supporting this

533 notion have been produced in bottlenose dolphins (e.g., Tursiops sp.), where eigenvector

534 centrality was found to predict survival in males but not females (Stanton \& Mann, 2012). On the

535 other hand, it is also possible that juvenile females were less central in the networks because we

536 only analyzed peer networks and therefore excluded interactions with adults (and infants).

537 Juvenile females may bias their social interactions (especially social grooming) to adult females

538 (Jarrett et al., 2018; Kulik et al., 2015; Widdig et al., 2015) and infants (as they practice

539 allomothering) in order to form the long-lasting social bonds that enhance individual (Brent et

540 al., 2017; Silk et al., 2010) and infant survival (Silk et al., 2003). Males may, on the other hand,

541 bias interactions to other juveniles, especially other males (Hassett, Rupp, \& Wallen, 2009) to

542 form relationships that can be beneficial during dispersal. Although we intentionally examined

543 peer networks, future studies should investigate overall network positions of developing

544 primates. In this case, we may see deviations in network integration between males and females,

545 with females potentially being more integrated into the overall adult network than males.

546 Similarly, we found that older juveniles were more central in grooming networks, indicating that 
547 juveniles have a developmental shift from play to grooming. As primates age, especially females,

548 they are more likely to focus on grooming interactions to solidify bonds, as play becomes less

549 and less frequent, which allows for successful integration into the group (Kulik et al., 2015).

550 In experiment 2, following removal from their mothers and the formation of the yearling

551 group, the predominant factor influencing network position across all three social networks

552 (social contact, social grooming, and social play) was early social experience. Although

553 dominance rank predicted the social contact eigenvector centrality, this could still have been

554 heavily influenced by early social experiences. We have previously demonstrated that early

555 social experiences predicted dominance rank, with MG outranking UG subjects after group

556 formation, and the opposite after group relocation (Wooddell et al., 2017a). Therefore, our

557 results suggest that dominance rank was less important in structuring social networks when in the

558 absence of the mother. While it could be argued that we would not expect to find a relationship

559 between social network position and dominance rank following relocation because the

560 dominance ranks were in constant flux and very unstable (Wooddell et al., 2017a) and therefore

561 network positions might be unstable too, this was not the case. Time period was not a predictor

562 (except in the social contact network), indicating that even during a period of stability,

563 dominance rank was not a predominant predictor of social network position in this population.

564 Secondly, individuals were stable in their social network position before and after relocation $\left(\mathrm{R}^{2}\right.$

565 ranged from 0.40 to 0.76 across the different social behaviors examined), suggesting that

566 although dominance ranks changed significantly, network positions did not. These findings

567 indicate that the early social experiences were crucial in determining network positions, and that

568 infants from highly complex, MG groups may have a specific advantage in forming social

569 relationships. The social connections made early in life are shaped by the early social 
570 environment (MG or UG), can remain stable in new environments, and can promote the

571 development of similar social networks later on, even in the absence of the mother (e.g., African

572 elephants, Loxodonta africana: Goldenberg et al., 2016). These stable relationships can be

573 especially important during times of social stress and instability and can act as a social buffer,

574 which is an area for future study.

575 In the naturalistic population, social contact and social play eigenvector values were

576 positively correlated for infants and juveniles, whereas in the peer group, social contact/social

577 grooming were inversely correlated with social play eigenvector values. We hypothesize that

578 social play may have been functionally different in the peer group than in the naturalistic group.

579 In the naturalistic field station, play may have been a primary source of affiliation, as dominance

580 ranks were primarily governed by maternal rank. In the peer group, while maternal rank did

581 predict dominance rank (see Wooddell et al., 2017a), this was true primarily only for the MG

582 subjects (indicating sustained maternal rank inheritance). Dominance ranks between UG-UG and

583 MG-UG subjects therefore depended on other extrinsic factors aside from maternal rank. Social

584 play may have functioned as not only an affiliative behavior, but also as a way to assess rivals

585 and practice skills necessary for fighting (as in yellow-bellied marmots, Marmota flaviventris:

586 Blumstein, Chung, \& Smith, 2013; and chimpanzees: Pan troglodytes: Paquette, 1994),

587 especially for UG-UG and MG-UG dyads. In doing so, individuals that were well-connected in

588 the social contact and social grooming networks were less connected in social play networks, as

589 social play may have been a precursor to aggressive interactions (Paquette, 1994). Social play

590 can likely have different social functions depending on the unique sociodynamics of the group.

591 Play can also have direct fitness outcomes such as preparing males for intrasexual competition 
592 for access to females, or in females for forming coalitions to defend resources and maintain the

593 social hierarchy.

594 Our study is not without its limitations. First, our highly skewed sex ratios, which were

595 beyond our control, in nearly every study group limited our ability to draw conclusions about sex

596 differences. Ideally, we would have a greater number of young females (and adult males) in

597 order to assess differences between sexes, as males and females have different developmental

598 trajectories (Barale et al., 2015; Kulik et al., 2015; Widdig et al., 2015) particularly as they

599 prepare for their sexually dimorphic adult social roles. Although we are limited in drawing

600 conclusions about sex differences, the greater number of immature male subjects is an

601 unintentional added benefit, as relatively less is known about juvenile male social networks in

602 female philopatric species. In addition, although our study was designed to assess the influence

603 of the mother's presence, we did this by examining groups with mothers and other kin compared

604 to a peer group without mothers and adult kin. Another way to examine the influence of the

605 mother, which would provide invaluable information about the direct impact of the mother's

606 presence on network development, would be to focus on constructing social networks in which

607 the mother is or is not nearby. This could add to the literature depicting the processes by which

608 infant networks mirror maternal networks. Indeed, previous research has indicated that mothers

609 can direct their offspring's interactions towards close kin (Berman et al., 1997) or non-

610 matrilineal group members (Timme, 1995), which can all influence the developmental trajectory

611 of infants' networks.

612 Dominance rank is a predominant factor in the structuring of adult social networks, and

613 here, we have also demonstrated substantial similar effects for infants and juveniles. Although

614 high-ranking immature primates are more central in their affiliative networks, this seems to be 
615 primarily present in multigenerational groups in which the mother is present, although the exact

616 mechanisms behind this process remain unknown. Multigenerational kin support is seen in a

617 number of long-lived animals, including African elephants (Shannon et al., 2013), orcas (Wright,

618 Stredulinksky, Ellis, \& Ford, 2016), primates (Berman, 1980; Berman et al., 1997), and humans

619 (Sear, Mace, \& McGregor, 2000). There may be a potential evolutionary advantage to having

620 complex family structures, as large extended families can provide leadership and knowledge that

621 aid in survival (Sear et al., 2000; Wright et al., 2016), but also possibly, sociality (Shannon et al.,

622 2013). Furthermore, our findings are also consistent with the human literature showing how the

623 presence of kin, such as grandmothers, plays a key role for infant rearing and development in

624 humans (Hawkes et al., 1998), hinting at the intriguing possibility of the evolutionary origin of

625 family support in the development of social skills. By examining the direct role that mothers and

626 kin play in shaping social networks, future research will shed light onto the role the mother and

627 other kin have in the behavioral developmental trajectory of immature offspring, which has long-

628 term fitness consequences.

\section{Acknowledgements}

631 Shriver National Institute of Child Health and Human Development, granted to Dr. Stephen J.

632 Suomi. The authors have no conflicts of interest to declare. We thank Dr. Suomi for his support

633 of this project. We are also grateful to Ashley M. Murphy for assistance in data collection in

634 experiment 2 and to the many interns and volunteers who assisted in data entry. We would also

635 like to thank Dr. Kevin Fuji for his assistance in data analysis. Finally, this work would not have

636 been possible without the wonderful animal care and veterinary staff at the NIH Animal Center,

637 who we are grateful for. 
638

639

640

641

642

643

644

645

646

647

648

649

650

651

652

653

654

655

656

657

658

659

\section{References}

Agostini, I., \& Visalberghi, E. (2005). Social influences on the acquisition of sex-typical

foraging patterns by juveniles in a group of wild tufted capuchin monkeys (Cebus nigritus). American Journal of Primatology, 65(4), 335-351. https://doi.org/10.1002/ajp.20120

Altmann J. (1974). Observational study of behavior: sampling methods. Behaviour, 49(3), 227 266. https://doi.org/10.1163/156853974X00534

Barale, C.L., Rubenstein, D.I., \& Beehner, J.C. (2015). Juvenile social relationships reflect adult patterns of behavior in wild geladas. American Journal of Primatology, 77(10), 10861096. https://doi.org/10.1002/ajp.22443

Berman, C.M. (1980). Early agonistic experience and rank acquisition among free-ranging infant rhesus monkeys. International Journal of Primatology, 1(2), 153-170. https://doi.org/10.1007/BF02735595

Berman, C.M. (1982). The ontogeny of social relationships with group companions among freeranging infant rhesus monkeys I. Social networks and differentiation. Animal Behaviour, 30(1), 149-162. https://doi.org/10.1016/S0003-3472(82)80250-9

Berman, C.M., Rasmussen, K.L.R., \& Suomi, S.J. (1997). Group size, infant development and social networks in free-ranging rhesus monkeys. Animal Behaviour, 53, 405-421. https://doi.org/10.1006/anbe.1996.0321

Blumstein, D. T., Chung, L. K. \& Smith, J. E. (2013). Early play may predict later dominance relationships in yellow-bellied marmots (Marmota flaviventris). Proc. R. Soc. Lond. [Biol] 280, 20130485. https://doi.org/10.1098/rspb.2013.0485 
660 Bonacich, P. (2007). Some unique properties of eigenvector centrality. Social Networks, 29(4),

661 555-564. https://doi.org/10.1016/j.socnet.2007.04.002

662 Borgeaud, C., \& Bshary, R. (2015). Wild vervet monkeys trade tolerance and specific coalitionary support for grooming in experimentally induced conflicts. Current Biology, 25(22), 3011-3016. https://doi.org/10.1016/j.cub.2015.10.016

665

666

667

668

669

670

671

672

673

674

675

676

677

678

679

680

681
Borgeaud, C., Sosa, S., Sueur, C., \& Bshary, R. (2017). The influence of demographic variation on social network stability in wild vervet monkeys. Animal Behaviour, 134, 155-165. https://doi.org/10.1016/j.anbehav.2017.09.028

Brent, L.J.N. (2015). Friends of friends: are indirect connections in social networks important to animal behaviour? Animal Behaviour, 103, 211-222. https://doi.org/10.1016/j.anbehav.2015.01.020

Brent, L.J.N., Heilbronner, S.R., Horvath,J.E., Gonzalez-Martinez, J., Ruiz-Lambides, A., Robinson, A.G., Pate Skene, J.H., \& Platt, M.L. (2013). Genetic origins of social networks in rhesus macaques. Scientific Reports, 3, 1042. https://doi.org/10.1038/srep01042

Brent, L.J.N., Ruiz-Lambides, A., \& Platt, M. L. (2017). Family network size and survival across the lifespan of female macaques. Proceedings. Biological sciences, 284(1854), 20170515. https://doi.org/10.1098/rspb.2017.0515

Burnham, K.P., Anderson, D.R., \& Huyvaert, K.P. (2011). AIC model selection and multimodel inference in behavioral ecology: some background, observations, and comparisons. Behavioral Ecology and Sociobiology, 65, 23-35. https://doi.org/10.1007/s00265-010$1029-6$ 
682 Carne, C., Wiper, S., \& Semple, S. (2011). Reciprocation and interchange of grooming, agonistic

683

684

685

686

687

688

689

690

691

692

693

694

695

696

697

698

699

700

701

702

703

704

support, feeding tolerance, and aggression in semi-free-ranging Barbary macaques. American Journal of Primatology, 73(11), 1127-1133. DOI 10.1002/ajp.20979

Cheney, D.L. (1977). The acquisition of rank and the development of reciprocal alliances among free-ranging immature baboons. Behavioral Ecology and Sociobiology, 2(3), 303-318. https://doi.org/10.1007/BF00299742

Coelho, C.G., Falótico, T., Izar, P., Mannu, M., Resende, B.D., Siqueira, J.O., \& Ottoni, E.B. (2015). Social learning strategies for nut-cracking by tufted capuchin monkeys (Sapajus spp.). Animal Cognition, 18(4), 911-919. https://doi.org/10.1007/s10071-015-0861-5

Cristol, D., \& Ketterson, E. (1990). Effect of prior residence on dominance status of dark-eyed juncos, Junco hyemalis. Animal Behaviour, 40, 580-586. https://doi.org/10.1016/S00033472(05)80539-1

de Ruiter, J.R.D., \& Geffen, E. (1998). Relatedness of matrilines, dispersing males and social groups in long-tailed macaques (Macaca fascicularis). Proceedings of the Royal Society of Britain, 265(1391), 79-87. https://doi.org/10.1098/rspb.1998.0267

Dettmer, A.M., Kaburu, S.S.K., Byers, K.L., Murphy, A.M., Soneson, E., Wooddell, L.J., \& Suomi, S.J. (2016). First-time rhesus monkey mothers, and mothers of sons, preferentially engage in face-to-face interactions with their infants. American Journal of Primatology, 78, 238-246. https://doi.org/10.1002/ajp.22503

Dettmer, A.M., Kaburu, S.S.K., Simpson, E.A., Paukner, A., Sclafani, V., Byers, K.L., Murphy, A.M., Miller, M., Marquez, N., Miller, G.M., Suomi, S.J., \& Ferrari, P.F. (2016). Neonatal face-to-face interactions promote later social behaviour in infant rhesus monkeys. Nature Communications, 7, 11940. https://doi.org/10.1038/ncomms11940 
705

706

707

708

709

710

711

712

713

714

715

716

717

718

719

720

721

722

723

724

725

726

727

Dunbar, R.I.M. (1991). Functional significance of social grooming in primates. Folia Primatologica, 57, 121-131. https://doi.org/10.1159/000156574

Farine, D. R. (2017). A guide to null models for animal social network analysis. Methods in ecology and evolution, 8(10), 1309-1320. doi: 10.1111/2041-210X.12772

Farine, D.R., \& Whitehead, H. (2015). Constructing, conducting and interpreting animal social network analysis. Journal of Animal Ecology, 84(5), 1144-1163. doi: 10.1111/13652656.12418

Fedurek, P., \& Lehmann, J. (2017). The effect of excluding juveniles on apparent adult olive baboons (Papio Anubis) social networks. PLoS ONE, 12(3), e0173146. https://doi.org/10.1371/journal.pone.0173146

Gerber, L., Krützen, M., de Ruiter, J.R., van Schaik, C.P., \& van Noordwijk, M.A. (2015). Postdisperal nepotism in male long-tailed macaques (Macaca fascicularis). Ecology and Evolution, 6(1), 46-55. https://doi.org/10.1002/ece3.1839

Goldenberg, S. Z., Douglas-Hamilton, I. \& Wittemyer, G. (2016). Vertical transmission of social roles drives resilience to poaching in elephant networks. Current Biology, 26, 75-79. https://doi.org/10.1016/j.cub.2015.11.005

Griffin, R.H., \& Nunn, C.L. (2011). Community structure and the spread of infectious disease in primate social networks. Evolutionary Ecology, 26(4), 779-800. doi: 10.1007/s10682011-9526-2

Handcock, M., Hunter, D., Butts, C., Goodreau, S., \& Morris, M. (2006). Statnet: an R package for the statistical analysis and simulation of social networks. University of Washington.

Harvey, P. H. \& Clutton-Brock, T. H. (1985). Life history variation in primates. Evolution, 39, 559-581. https://doi.org/10.1111/j.1558-5646.1985.tb00395.x 
728 Hassett, J.M., Rupp, H.A., \& Wallen, K. (2009). Social segregation in male, but not female 729 yearling rhesus macaques (Macaca mulatta). American Journal of Primatology, 72(2), 87-92. https://doi.org/10.1002/ajp.20756

731 Hauser, M.D. (1988). How infant vervet monkeys learn to recognise starling alarm calls: the role of experience. Behaviour, 105, 187-201. http://dx.doi.org/10.1163/156853988X00016

733 Hawkes, K., O’Connell, J.F., Blurton Jones, N.G., Alvarez, H., \& Charnov, E.L. (1998).

734 Grandmothering, menopause, and the evolution of human life histories. Proceedings of the National Academy of Sciences 95(3), 1336-1339.

737 Hobaiter, C., Poisot, T., Zuberbühler, K., Hoppitt, W., \& Gruber, T. (2014). Social Network

$738 \quad$ Analysis Shows Direct Evidence for Social Transmission of Tool Use in Wild Chimpanzees. PLoS Biology, 12(9), e1001960.

741 Ilany, A., \& Akçay, E. (2016). Social inheritance can explain the structure of animal social networks. Nature Communications, 7, 12084. https://doi.org/10.1038/ncomms12084

743 Janson, C. H. \& van Schaik, C. P. (1993). Ecological risk aversion in juvenile primates: slow and steady wins the race. In (M. Pereira \& L. Fairbanks, Eds) Juvenile Primates, pp. 62-73.

746 Jarrett, J.D., Bonnell, T.R., Young, C., Barrett, L., \& Henzi, S.P. (2018). Network integration and limits to social inheritance in vervet monkeys. Proceedings of the Royal Society of Britain: Biological Sciences, 285(1876), 20172668. https://doi.org/10.1098/rspb.2017.2668 
750 Joffe, T.H. (1997). Social pressures have selected for an extended juvenile period in primates.

751 Journal of Human Evolution, 32(6), 593-605. https://doi.org/10.1006/jhev.1997.0140

752 Kappeler, P. M., \& van Schaik, C. P. (2002). Evolution of primate social systems. International 753 Journal of Primatology, 23(4), 707-740. https://doi.org/10.1023/A:1015520830318

754 Kasper, C., \& Voelkl, B. (2009). A social network analysis of primate groups. Primates, 50(4), 755 343-356. doi: 10.1007/s10329-009-0153-2

756 Koivula, K., Lahti, K., Orell, M., \& Rytkönen., S. (1993). Prior residency as a key determinant 757 of social dominance in the willow tit (Parus montanus). Behavioral Ecology and $758 \quad$ Sociobiology, 33(4), 283-287. https://doi.org/10.1007/BF02027126

759 Kroenke, C.H., Kubzansky, L.D., Schernhammer, E.S., Holmes, M.D., \& Kawachi, I. (2006). 760 Social networks, social support, and survival after breast cancer diagnosis. Journal of 761 Clinical Oncology, 24(7), 1105-1111. DOI: 10.1200/JCO.2005.04.2846

762 Kulik, L., Amici, F., Langos, D., \& Widdig, A. (2015). Sex differences in the development of social relationships in rhesus macaques (Macaca mulatta). International Journal of Primatology, 36(2), 353-376. https://doi.org/10.1007/s10764-015-9826-4

Lehmann, J., Korstjens, A.H., \& Dunbar, R.I.M. (2007). Group size, grooming, and social cohesion in primates. Animal Behaviour, 74(6), 1617-1629. survival of wild Barbary macaques, Macaca Sylvanus. Behavioral Ecology, 27(1), 20-28. https://doi.org/10.1093/beheco/arv169 
771 Lonsdorf, E.V. (2017). Sex differences in nonhuman primate behavioral development. Journal of $772 \quad$ Neuroscience Research, 95(1-2), 213-221. https://doi.org/10.1002/jnr.23862

773 MacIntosh, A.J.J., Jacobs, A., Garcia, C., Shimizu, K., Mouri, K., Huffman, M.A., et al. (2012) 774 Monkeys in the Middle: Parasite Transmission through the Social Network of a Wild 775 Primate. PLoS ONE 7(12), e51144. https://doi.org/10.1371/journal.pone.0051144

776

777

778

779

780

781

782

783

784

785

786

787

788

789

790

791

Maestripieri, D. (2018). Maternal influences on primate social development. Behavioral Ecology and Sociobiology, 72, 130. https://doi.org/10.1007/s00265-018-2547-x

McCowan, B., Anderson, K., Heagarty, A., \& Cameron, A. (2008). Utility of social network analysis for primate behavioral management and well-being. Applied Animal Behaviour Science, 109(2-4), 396-405. https://doi.org/10.1016/j.applanim.2007.02.009

McCowan, B., Beisner, B., Bliss-Moreau, E., Vandeleest, J., Jin, J., Hannibal, D., \& Hsieh, F. (2016). Connections matter: social networks and lifespan health in primate translational models. Frontiers in Psychology, 7, 433. https://doi.org/10.3389/fpsyg.2016.00433

Murray, C.M., Lonsdorf, E.V., Stanton, M.A., Wellens, K.R., Miller, J.A., Goodall, J., \& Pusey, A.E. (2014). Early social exposure in wild chimpanzees: mothers with sons are more gregarious than mothers with daughters. Proc Natl Acad Sci USA, 111, 18189-18194. https://doi.org/10.1073/pnas.1409507111

Neumann, C., Duboscq, J., Dubuc, C., Ginting, A., Maulana Irwan, A., Agil, M., Widdig, A., \& Engelhardt, A. (2011). Assessing dominance hierarchies: validation and advantages of progressive evaluation with Elo-rating. Animal Behaviour, 82, 911-921.

DOI:10.1016/j.anbehav.2011.07.016 
792 Newman, M.E.J. (2004). Analysis of weighted networks. Physical Review E, 69(2), 026113.

$793 \quad$ https://doi.org/10.1103/PhysRevE.70.056131

794 Novak, M., Kinsey, J., Jorgensen, M., Hazen, T. (1998). Effects of puzzle feeders on

795 pathological behavior in individually housed rhesus monkeys. American Journal of

796

797 Primatology. 46(3), 213-27. https://doi.org/10.1002/(SICI)10982345(1998)46:3<213::AID-AJP3>3.0.CO;2-L

798

799

800

801

802

803

804

805

806

807

808

809

810

811

812

813

814

Palagi, E. (2018). Not just for fun! Social play as a springboard for adult social competence in human and non-human primates. Behavioral Ecology and Sociobiology, 72(6), 90. https://doi.org/10.1007/s00265-018-2506-6

Paquette, D. (1994). Fighting and playfighting in captive adolescent chimpanzees. Aggressive Behavior, 20, 49-65. https://doi.org/10.1002/1098-2337(1994)20:1<49::AIDAB2480200107>3.0.CO;2-C

Pellis, S. M., Burghardt, G. M., Palagi, E. \& Mangel, M. (2015). Modeling play: Distinguishing between origins and current functions. Adapt. Behavi. 1059712315596053. https://doi.org/10.1177/1059712315596053

Pereira, M.E., \& Fairbanks, L.A. (Eds.), (2002). Juvenile primates: Life history, development, and behavior, Oxford University Press, New York, NY.

Pinter-Wollman, N., Hobson, E.A., Smith, J.E., Edelman, A.J., Shizuka, D., de Silva, S., Waters, J.S., Prager, S.D., Sasaki, T., Wittemyer, G., Fewell, J., \& McDonald, D.B. (2014). The dynamics of animal social networks: analytical, conceptual, and theoretical advances. Behavioral Ecology, 25(2), 242-255. https://doi.org/10.1093/beheco/art047

Poirier, F.E., \& Smith, E.O. (1974). Socializing functions of primate play. American Zoologist. 14(1), 275-287. https://doi.org/10.1093/icb/14.1.275 
815 Ramakrishnan, U., \& Coss, R.G. (2001). Age differences in the responses to adult and juvenile 816 alarm calls by bonnet macaques (Macaca radiata). Ethology, 106(2), 131-144. https://doi.org/10.1046/j.1439-0310.2000.00501.x

818 Rapaport, L.G., \& Brown, G.R. (2008). Social influences on foraging behavior in young nonhuman primates: Learning what, where, and how to eat. Evolutionary Anthropology, 17(4), 189-201. https://doi.org/10.1002/evan.20180

821 Ross, C., \& Jones, K.E. (1999). Socioecology and the evolution of primate reproductive rates. In: Lee PC, editor. Comparative Primate Socioecology. New York: Cambridge University Press. $p$ 73-110.

Rushmore, J., Caillaud, D., Matamba, L., Stumpf, R.M., Borgatti, S.P., \& Altizer, S. (2013). Social network analysis of wild chimpanzees provides insights for predicting infectious

827 Sade, D.S., Altmann, M., Loy, J., Hausfater, G., \& Breuggeman, J.A. (1988). Sociometrics of Macaca mulatta: II. Decoupling centrality and dominance in rhesus monkey social networks. American Journal of Physical Anthropology, 77, 409-425.

Schino, G. (2007). Grooming and agonistic support: a meta-analysis of primate reciprocal altruism. Behavioral Ecology, 18(1), 115-120. https://doi.org/10.1098/rsbl.2007.0506

833 Schülke, O., Bhagavatula, J., Vigilant, L., \& Ostner, J. (2010). Social bonds enhance reproductive success in male macaques. Current Biology, 20(24), 2207-2210. https://doi.org/10.1016/j.cub.2010.10.058 Theoretical Biology, 65(4), 671-698. https://doi.org/10.1016/0022-5193(77)90015-7 
838 Seyfarth, R.M. Cheney, D.L., \& Marler, P. (1980). Monkey responses to three different alarm

839 calls: evidence of predator classification and semantic communication. Science, 210(4471), 801-803. DOI: 10.1126/science.7433999

841 Shannon, G., Slotow, R., Durant, S.M., Sayialel, K.N., Poole, J., Moss, C., \& McComb, K. (2013). Effects of social disruption in elephants persists decades after culling. Frontiers in Zoology, 10(62). https://doi.org/10.1186/1742-9994-10-62

844 Shimada, M., \& Sueur, C. (2014). The importance of social play network for infant or juvenile wild chimpanzees at Mahale Mountains National Park, Tanzania. American Journal of Primatology, 76(11), 1025-1036. https://doi.org/10.1002/ajp.22289

847 Silk, J.B., Alberts, S.C., \& Altmann, J. (2003). Social bonds of female baboons enhance infant survival. Science, 302(5648), 1231-1234. DOI: 10.1126/science.1088580

Silk, J.B., Beehner, J.C., Bergman, T.J., Crockford, C., Engh, A.L., Moscovice, L.R., Wittig, enhance the longevity of female baboons. Current Biology, 20(5), 1359-1361. https://doi.org/10.1016/j.cub.2010.05.067

Smith, K.P., \& Christakis, N.A. (2008). Social networks and health. Annual Review of Sociology, 34, 405-429. https://doi.org/10.1146/annurev.soc.34.040507.134601 Social status drives social relationships in groups of unrelated female rhesus macaques.

858 Stanton, M.A. \& Mann, J. (2012). Early social networks predict survival in wild bottlenose dolphins. PLoS ONE, 7(10), e47508. https://doi.org/10.1371/journal.pone.0047508 
860 Sueur, C., Jacobs, A., Amblard, F., Petit, O., \& King, A.J. (2011). How can social network

861

862

863

864

865

866

867

868

869

870

871

872

873

874 Turner, J.W., Bills, P.S., \& Holekamp, K.E. (2018). Ontogenetic change in determinants of

875

876

877 Van de Waal, E., Claidiére, N., \& Whiten, A. (2013). Social learning and spread of alternative

878

879 analysis improve the study of primate behavior? American Journal of Primatology, 73(8), 703-719. https://doi.org/10.1002/ajp.20915

Sueur, C., \& Petit, O. (2008). Organization of group members at departure is driven by social structure in Macaca. International Journal of Primatology, 29(4), 1085-1098. https://doi.org/10.1007/s10764-008-9262-9

Sueur, C., Petit, O., de Marco, A., Jacobs, A.T., Watanabe, K., \& Thierry, B. (2011). A comparative network analysis of social style in macaques. Animal Behaviour, 82, 845852. https://doi.org/10.1016/j.anbehav.2011.07.020

Thierry, B. (2007). Unity in diversity: lessons from macaque societies. Evolutionary Anthropology, 16, 224-238.

Timme, A. (1995). Sex differences in infant integration in a semifree-ranging group of Barbary macaques (Macaca sylvanus, L. 1758) at Salem, Germany. American Journal of Primatology, 37, 221-231. https://doi.org/10.1002/ajp.1350370304 social network position in the spotted hyena. Behavioral Ecology and Sociobiology, 72(10). https://doi.org/10.1007/s00265-017-2426-x means of opening an artificial fruit in four groups of vervet monkeys. Animal Behaviour, 85(1), 71-76. https://doi.org/10.1016/j.anbehav.2012.10.008 
880

881

882

883

884

885

886

887

888

889

890

891

892

893

894

895

896

897

898

899

900

901

902

Van Noordwijk, M.A., \& van Schaik, C.P. (2001). Career moves: transfer and rank challenge decisions by male long-tailed macaques. Behaviour, 138(3), 359-395. https://doi.org/10.1163/15685390152032505

Vandeleest, J.J., Beisner, B.A., Hannibal, D.L., Nathman, A.C., Capitanio, J.P., Hsieh, F., Atwill, E.R., \& McCowan, B. (2016). Decoupling social status and status certainty effects on health in macaques: a network approach. PeerJ(4), e2394. DOI: 10.7717/peerj.2394

Vogt, T.M., Mullooly, J.P., Ernst, D., Pope, C.R., \& Hollis, J.F. (1992). Social networks as predictors of ischemic heart disease, cancer, stroke, and hypertension: incidence, survival and mortality. Journal of Clinical Epidemiology, 45(6), 659-666. https://doi.org/10.1016/0895-4356(92)90138-D

Widdig, A., Langos, D., \& Kulik, L. (2015). Sex differences in kin bias at maturation: Male rhesus macaques prefer paternal kin prior to natal dispersal. American Journal of Primatology, 78(1), 78-91. https://doi.org/10.1002/ajp.22401

Williams, R., \& Lusseau, D. (2006). A killer whale social network is vulnerable to targeted removals. Biology Letters, 2, 497-500. https://doi.org/10.1098/rsbl.2006.0510

Wooddell, L.J., Kaburu, S.S.K., Murphy, A.M., Suomi, S.J., \& Dettmer, A.M. (2017a). Rank acquisition in rhesus macaque yearlings following permanent maternal separation: The importance of the social and physical environment. Developmental Psychobiology, 59(7): 863-875. https://doi.org/10.1002/dev.21555

Wooddell, L.J., Kaburu, S.S.K., Suomi, S.J., Dettmer, A.M. (2017b). Elo-rating for tracking rank fluctuations following demographic changes involving semi-free ranging rhesus macaques (Macaca mulatta). Journal of the American Assocation for Laboratory Animal Science, 56(3), 1-9. 
903 Wright, B.M., Stredulinksky, E.H., Ellis, G.M., \& Ford, J.K.B. (2016). Kin-directed food sharing 904 promotes lifetime natal philopatry of both sexes in a population of fish-eating killer 905 whales, Orcinus orca. Animal Behaviour, 115, 81-95.

906 https://doi.org/10.1016/j.anbehav.2016.02.025

907 Young, C., Majolo, B., Heistermann, M., Schülke, O., \& Ostner, J. (2014). Responses to social 908 and environmental stress are attenuated by strong male bonds in wild macaques.

909 Proceedings of the National Academy of Sciences of the United States of America, 910 111(51), 18195-18200. https://doi.org/10.1073/pnas.1411450111

911

912

913

914

915

916

917

918

919

920

921

922

923

924

925 


\section{$926 \quad$ Figure Legends}

927 Figure 1: Social networks in adults (A), juveniles (B), and infants (C) in a naturalistic population of rhesus macaques

The adult social grooming network (A), juvenile social play network (B) and infant social play $(\mathrm{C})$ networks in a group of semi-free ranging rhesus macaques. Nodes represent individuals, while the numbers inside the nodes represent individuals' ordinal ranks, with 1 being the highest-ranking individual. Node sizes are scaled based on eigenvector centrality with larger nodes representing more socially connected individuals. Lines between individuals represent interactions among one another. Shapes refer to sex (squares are males, circles are females). Colors represent matrilines, and matrilines are ranked in order of dominance rank: 3 (blue; dominant matriline), 4 (red; intermediate ranking matriline), and 1 (yellow; lowest-ranking matriline). Notably, the alpha male is from matriline 1, but is the highest-ranking animal. Across all ages, high-ranking individuals are more central in their peer social networks. Mothers' eigenvector centrality in the social grooming network predicted offspring's

943 Figure 3: Juvenile male centrality in social networks play networks. 


\section{Table Legends}

950

951 Table 1. Results of the regression models testing whether Elo-rating values, sex, age and the 952 interaction between sex and Elo-rating significantly predicted adult, juvenile and infant social 953 contact, social grooming, and social play eigenvector values. Only the results from the best 954 model are presented.

955

956 Table 2. Results of the LMM models testing whether mothers' grooming eigenvector, infant sex, 957 and their interaction significantly predicted their offspring's play eigenvector. Only the results 958 from the best model are presented.

959

960 Table 3. Results of the LMM models ran to test whether yearling early social experience, Elo961 rating, period (before vs after relocation), sex, and age predicted their eigenvector values. Only 962 the results from the best model are presented.

964 Supplementary Material:

965 Comparison of observed vs randomized networks in rhesus macaques: All networks 966 generated from real social data were significantly different from randomized networks. 